\title{
Expression of Mammaglobin and Carcinoembryonic Antigen in Peripheral Blood of Patients with Breast Cancer Using Real Time Polymerase Chain Reaction
}

\author{
Negin Khosravi' ${ }^{1}$ Naghmeh Bahrami ${ }^{2,3,4}$, Adnan Khosravi' ${ }^{5}$ Atefeh Abedini', Arda Kiani6, \\ Somayeh Sharifynia7 ${ }^{7}$, Pegah Gharaeeyan ${ }^{1}$, Sharareh Seifi ${ }^{8}$, Abdolreza Mohamadnia ${ }^{9,10^{*}}$ \\ ${ }^{1}$ Islamic Azad University, Tehran, Iran \\ ${ }^{2}$ Craniomaxillofacial Research Center, Tehran University of Medical Sciences, Tehran, Iran \\ ${ }^{3}$ Oral and Maxillofacial Surgery Department, School of Dentistry, Tehran University of Medical Sciences, Tehran, Iran \\ ${ }^{4}$ Iranian Tissue Bank \& Research Center, Tehran University of Medical Sciences, Tehran, Iran \\ ${ }^{5}$ Tobacco Prevention and Control Research Center, National Research Institute of Tuberculosis and Lung Diseases (NRITLD), \\ Shahid Beheshti University of Medical Sciences, Tehran, Iran \\ ${ }^{6}$ Tracheal Diseases Research Center, National Research Institute of Tuberculosis and Lung Diseases (NRITLD), Shahid Beheshti \\ University of Medical Sciences, Tehran, Iran \\ ${ }^{7}$ Clinical Tuberculosis and Epidemiology Research Center, National Research Institute of Tuberculosis and Lung Diseases \\ (NRITLD), Shahid Beheshti University of Medical Sciences, Tehran, Iran \\ ${ }^{8}$ Chronic Respiratory Diseases Research Center, National Research Institute of Tuberculosis and Lung Diseases (NRITLD), \\ Shahid Beheshti University of Medical Sciences, Tehran, Iran \\ ${ }^{9}$ Virology Research Center, National Research Institute of Tuberculosis and Lung Diseases (NRITLD), Shahid Beheshti University \\ of Medical Sciences, Tehran, Iran \\ ${ }^{10}$ Department of Biotechnology, School of Advanced Technologies in Medicine, Shahid Beheshti University of Medical Sciences, \\ Tehran, Iran \\ Email: *mohamadnia.ar@gmail.com
}

How to cite this paper: Khosravi, N., Bahrami, N., Khosravi, A., Abedini, A., Kiani, A., Sharifynia, S., Gharaeeyan, P., Seifi, S. and Mohamadnia, A. (2017) Expression of Mammaglobin and Carcinoembryonic Antigen in Peripheral Blood of Patients with Breast Cancer Using Real Time Polymerase Chain Reaction. Open Journal of Clinical Diagnostics, 7, 103-112.

https://doi.org/10.4236/ojcd.2017.74011

Received: November 1, 2017

Accepted: November 28, 2017

Published: December 1, 2017

\begin{abstract}
Background and Objectives: Breast cancer is among the most common causes of cancer related mortality in women worldwide. Early detection and prompt diagnosis of tumor is the first step to prevent cancer-related morbidity and mortality, and a comprehensive understanding of the involved molecular mechanisms can greatly help in this respect. Breast cancer, like many other types of cancer, is caused by a combination of genetic and epigenetic changes such as inactivation of tumor suppressor genes. Materials and Methods: This study was performed on 40 breast cancer patients and 40 healthy controls. Quantitative real time reverse transcription polymerase chain reaction (real time qRT-PCR) was used to assess the expression of carcinoembryonic antigen (CEA) and mammaglobin mRNA in the peripheral blood of
\end{abstract}


Copyright (C) 2017 by authors and Scientific Research Publishing Inc. This work is licensed under the Creative Commons Attribution International License (CC BY 4.0).

http://creativecommons.org/licenses/by/4.0/ patients and healthy controls. The two groups were compared using t-test. Results: The two groups were not significantly different in terms of the mean age. Twenty-nine out of 40 cancer patients were positive for CEA mRNA and its sensitivity was calculated to be $72.5 \%$. Twelve out of 40 healthy controls were positive for CEA mRNA. Twenty-six out of 40 patients were positive for mammaglobin mRNA indicative of $65 \%$ sensitivity while only five out of 40 healthy controls were positive for mammaglobin mRNA. Conclusion: Both CEA and mammaglobin mRNA had high sensitivity in cancer patients; thus, they can be used for screening and early detection of breast cancer patients. Further studies with larger sample sizes are required to confirm the current findings.

\section{Keywords}

Breast Cancer, Biomarker, Mammaglobin mRNA, Carcinoembryonic Antigen mRNA

\section{Introduction}

Breast cancer is a common malignancy in women. It is the first cause of cancerrelated mortality in women aged 40 to 55 years. It accounts for $18 \%$ of cancers in women and its prevalence in western countries is much higher than that in eastern countries [1]. Malignant breast tumors originate from mammary cells and involve one or both breasts. Breast cancer is among the most common causes of death due to cancer among women with approximately one million new cases annually worldwide [2]. Breast cancer is the result of unlimited growth and proliferation of epithelial cells lining the mammary ducts or lobules and may occur in various parts of the breast. It may develop in different tissues such as the lactiferous ducts, lactating cells or the parenchyma. Breast cancer is among the most common conditions affecting women worldwide [3]. Geographical differences noticed in the prevalence of breast cancer indicate the important role of environmental factors as risk factors for development of breast cancer. Westernized life style is believed to be responsible for increasing prevalence of breast cancer in developing countries, in women in Eastern Asia and Asian women residing in the United States [4]. Similar to other epithelial malignancies, the incidence of breast cancer increases with age and almost three-fourths of the cases of breast cancer occur in women older than 50 years. Higher occurrence in older ages negatively affects all phases from diagnosis to outcome and even the success rate of treatment [5]. Although breast cancer is much more common among women, it also occurs in men [6].

Tumor is defined as abnormal growth and proliferation of cells followed by cellular accumulation. In terms of growth and proliferation, tumors are divided into two groups of benign and malignant [7]. Metastasis is referred to as dissemination of malignant tumors by directly invading the adjacent tissues and formation of secondary tumors [8]. If diagnosed early, breast cancer patients would 
have a greater chance of recovery with simpler therapeutic strategies and lower intensity of treatment with $98 \%$ survival rate [9]. However, diagnosis after metastasis decreases the survival rate to $27 \%$ [10]; this highlights the importance of early detection of breast cancer, which depends on sensitive screening methods. In other words, detection of breast cancer in early stages is the key to recovery [9]. In the recent years, improved diagnostic modalities have enabled earlier detection of breast cancer and resulted in a reduction in the rate of metastasis and related mortality. However, high prevalence of breast cancer indicates the need for more efficient diagnostic and therapeutic strategies [10]. New diagnostic markers for early detection of breast cancer can greatly help in this respect and can even enable the oncologists to schedule an effective therapeutic strategy. The efficacy of several markers for detection of breast cancer has been evaluated in previous studies; however, only a few were accurate enough for application in the clinical setting [9] [11]. The characteristics of biomarkers must be detectable and measurable as their function and value. Biomarkers are measurable and are involved in functions of normal cells as well as the pathological processes or response to drugs [12]. Biomarkers may be used as the key molecules for detection of metabolic pathways, signaling and detection of stage of disease [13] [14] [15].

The CEA is a glycoprotein antigen present on the cell surface. It is produced in high amounts in human colon cancer; thus, its function and correlation with the occurrence of other types of cancers must be evaluated [16]. It has been reported to be an important biomarker for detection of colon cancer and some other types of cancers. The proteins encoded by the CEA-subgroup genes are present in cell membrane and their expression shows a complex pattern in healthy and cancerous tissues. Proteins coded by a couple of genes in the CEA family are located on the cell surface and have adhesion properties while some others are part of bile glycoproteins [16] [17].

The mammaglobin gene codes a secretory protein, which is a glycoprotein with $101 \mathrm{KD}$ molecular weight. In humans, the expression of this gene is limited to the mammary glands [18] [19]. Use of mammaglobin gene as a molecular marker for clinical diagnosis of primary and metastatic breast cancer has been recently suggested. Expression of mammaglobin gene mRNA was noted in $60 \%$ of peripheral stem cells of breast cancer patients [20] [21].

Considering all the above, this study sought to assess the expression of CEA and mammaglobin mRNA in the peripheral blood of patients with early-stage breast cancer. Early detection leads to early treatment and yields more favorable results and fewer complications compared to late diagnosis. The efficacy of other markers must also be evaluated to find a collection of biomarkers for accurate and early detection of breast cancer.

\section{Materials and Methods}

This study was approved by the ethics committee of Masih Daneshvari Hopsital, affiliated to Shahid Beheshti University of Medical Sciences. After obtaining 
ethical code to No.IR.SBMU.NRITLD.REC.1394.149, Forty breast cancer patients presenting with a definite diagnosis were randomly selected. Forty healthy controls were selected among those presenting for routine medical examinations.

Control subjects were confirmed to be cancer free following routine breast examinations.

The patient group included breast cancer patients in stages 1 - 3 without distant metastasis. The patients had not undergone any medical (chemotherapy or radiotherapy) or surgical intervention. Breast cancer had been pathologically confirmed. The patients and control subjects were matched in terms of age range.

Collection of peripheral blood sample:

A total of $12 \mathrm{~mL}$ of peripheral blood was obtained from all participants; the first $2 \mathrm{~mL}$ was discarded due to possible contamination with epithelial cells. The samples were immediately transferred to a laboratory for RNA extraction. This volume of peripheral blood was calculated based on the sensitivity required for real time qRT-PCR.

\subsection{RNA Extraction Using RNeasy Midi Kit}

RNA extraction was done using RNeasy Midi Kit (Qiagen Cat no. 75144). First, red blood cells were lysed using a lysing buffer, centrifuged and the obtained cell mass was rinsed with phosphate buffered saline twice and entered into the extraction phase. The obtained total RNA from each column was dissolved in the storage buffer provided in the kit. Pelleted leukocytes were first lysed using the solution available in the kit and the lysate was then homogenized. Ethanol was added and the lysate and the sediment (if present) were transferred to RNeasy Midi column for attachment of RNA to the column. The column was rinsed to eliminate excess materials and the extracted RNA was eluted. The extracted RNA was quantitatively and qualitatively analyzed using NanoDrop.

\subsection{Synthesis of cDNA}

The cDNA was synthesized using Viva 2-step RT-PCR Kit (Cat No. RTPL12). Of each RNA sample, three cDNA vials were synthesized and their quality was controlled by NanoDrop; $15 \mathrm{~mL}$ of the RNA was used for the synthesis of cDNA. The synthesized $\mathrm{cDNAs}$ were stored at $-80^{\circ} \mathrm{C}$ until the conduction of real time RT-PCR.

\subsection{Primer Designing}

Specific primers for each marker were designed using AlleleD6 software and ordered for synthesis. Table 1 presents the primers and their amount for use in the final reaction of real time-PCR.

\subsection{Real time RT-PCR}

Real-time RT-PCR was performed using HotTaq EvaGreen qPCR Mix (Cat. No. 
Table 1. Characteristics of the primers used in real time RT-PCR.

\begin{tabular}{cccc}
\hline & \multicolumn{2}{c}{ Gene } & \\
\cline { 2 - 4 } & CEA & Mammaglobin & 18 s rRNA \\
\hline F initiator & ACCCTGGATGTCCTCTATGG & TGCCATAGATGAATTGAAGGAATG & GTAACCCGTTGAACCCCATT \\
Primer length & 20 & 24 & 20 \\
R initiator & CAGGCATAGGTCCCGTTATTA & TGTCATATATTAATTGCATAAACACCTCA & CCATCCAATCGGTAGTAGCG \\
$\begin{array}{c}\text { Primer length } \\
\text { Length of amplified } \\
\text { segment }\end{array}$ & 21 & 29 & 20 \\
$\begin{array}{c}\text { Optimal annealing } \\
\text { temperature }\end{array}$ & 174 & 89 & 152 \\
\hline
\end{tabular}

BT11101). The RT-PCR reaction components included:

a) $2 \mu \mathrm{L}$ of template sequence

b) $4 \mu \mathrm{L}$ of Master mix

c) Primer based on the most suitable concentration found in primary set up tests

d) Deionized distilled water to reach a final reaction volume of $20 \mu \mathrm{L}$.

\subsection{Statistical Analysis}

Sample size was calculated according to the ratio of positivity of markers in the two groups estimated primarily based on similar previous studies [22] and taking into account type 1 error of 5\% and type 2 error of $20 \%$. The data were statistically analyzed using SPSS version 22 . The mean values were compared between the patient and control groups using t-test. Chi square test was applied to analyze the ratio of gene expression in the two groups. $\mathrm{P} \leq 0.05$ was considered statistically significant.

\section{Results}

The study was conducted on 40 patients with breast cancer and 40 healthy controls. The mean age was $51.60 \pm 14.46$ years in patients and $49.12 \pm 13.56$ years in controls. According to t-test, the difference in the mean age was not significant between the two groups $(\mathrm{P}=0.539)$.

In this study, $18 \mathrm{~s}$ rRNA gene was selected as the reference gene and studied in the two groups. Expression of this gene was relatively measured by real-time RT-PCR using ct value for each sample. The results showed no significant difference in this respect between the two groups, which confirmed the accuracy of its selection as the reference gene $(\mathrm{P}=0.239)$.

\subsection{Expression of CEA mRNA and Mammaglobin mRNA}

After data extraction, number of subjects with positive expression of the markers was determined. Among breast cancer patients, 29 out of 40 were positive for CEA mRNA; thus, the sensitivity of this marker was found to be $72.5 \%$. Among 


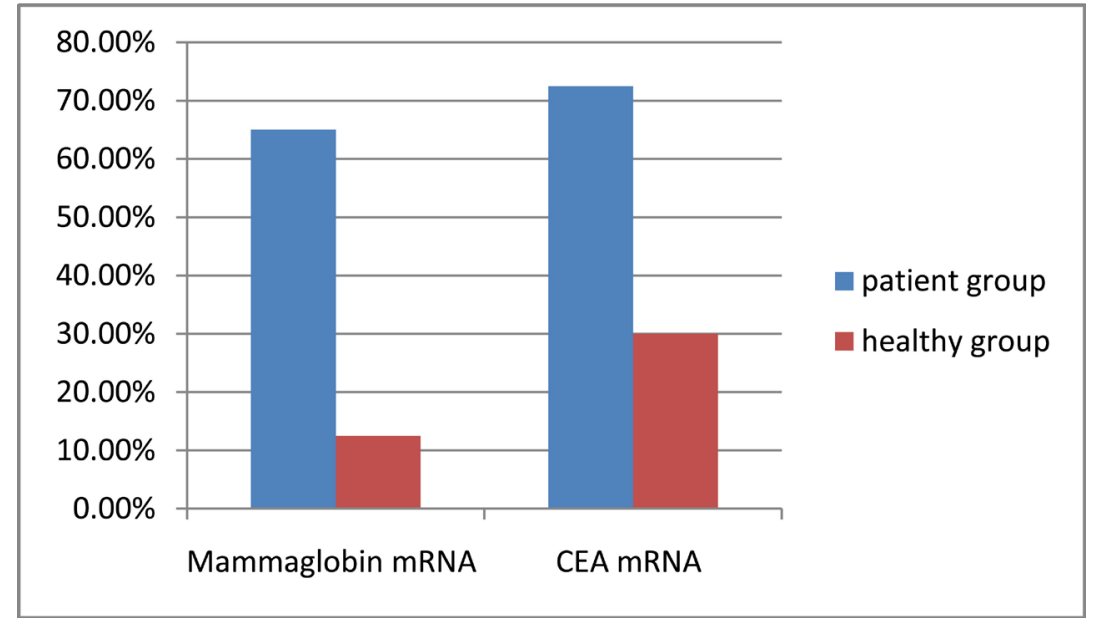

Figure 1. The expression levels of MUC1 mRNA and CEA mRNA in peripheral blood from NSCLC patients and healthy group.

healthy controls, 12 out of 40 were positive for CEA mRNA indicative of 30\% false positive results; thus, the specificity of this marker was calculated to be $70 \%$. The difference in this respect between the patient and control groups was statistically significant $(\mathrm{P}=0.003)$.

Among breast cancer patients, 26 out of 40 were positive for mammaglobin mRNA indicating $65 \%$ sensitivity. Among healthy controls, five out of 40 were positive for this marker indicating $12.5 \%$ false positive results. Thus, specificity was calculated to be $87.5 \%$. The difference between patients and controls in this respect was statistically significant $(\mathrm{P}=0.004)$ (Figure 1 ).

\subsection{Assessment of Difference in Expression of Markers in Patients and Controls}

The relative difference in expression of CEA mRNA and mammaglobin mRNA was measured between patients and healthy controls using $\Delta \Delta \mathrm{Ct}$ method.

Using $2^{-\Delta \Delta C t}$ formula, it was revealed that number of primary transcripts of this marker in patients was averagely 1.34 times the value in healthy controls and Mammaglobin mRNA was revealed that number of primary transcripts of this marker in patients was averagely 1.82 times the value in healthy controls (Figure 2).

\section{Discussion}

Cancer is a non-communicable chronic disease with not only physical but also psychological and socioeconomic impacts. Cancer occurs following uncontrollable cell proliferation as the result of environmental and genetic factors [4].

Breast cancer is among the most common malignancies in women and is the first cause of cancer related mortality in women aged 40 to 55 years. Breast cancer accounts for approximately $18 \%$ of all cancers in women with a much higher prevalence in western compared to eastern countries [1]. Breast cancer is the result of unlimited proliferation of epithelial cells lining the mammary ducts or 


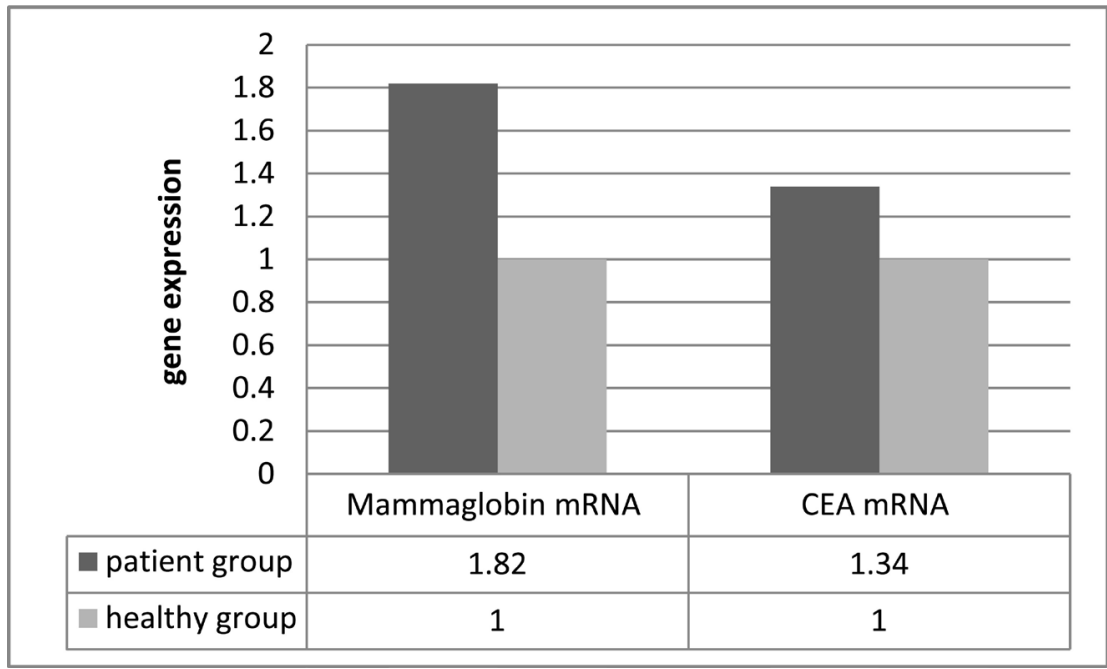

Figure 2. Difference in expression of MUC1 mRNA and CEA mRNA in peripheral blood from NSCLC patients and healthy group.

lobules and can occur in different parts of the breasts. It can involve ductal cells, lactating cells or parenchymal cells. Breast cancer is among the most common diseases in women worldwide [3] [23]. Annually, a large number of patients are diagnosed with breast cancer, many of which expire due to this condition. Breast cancer is an important epidemiological dilemma worldwide. Despite numerous advances in early detection and prompt treatment of breast cancer, it is still one of the most important causes of death due to cancer among women [3]. If diagnosed early, breast cancer would have a higher chance of recovery with simpler therapeutic strategies and less intense treatment yielding a survival rate of $98 \%$ [9]. This highlights the importance of early detection of breast cancer, which depends on sensitive and effective screening methods. In other words, early diagnosis of breast cancer is the key to recovery and favorable outcome [24]. Biomarkers are measurable factors, which are involved in activities of normal cells, pathological processes or response to drugs [12]. RNA markers have relatively optimal properties for use as diagnostic tests; thus, this group of biomarkers was selected for this study. On the other hand, they can be detected even in very small amounts using real-time RT-PCR, which has high sensitivity and specificity. This is an advantage of these markers compared to protein markers, which are often not detectable in very small amounts [25] [26] [27] [28]. In a study by Watson and Fleming on mammaglobin, it was shown that $80 \%$ of primary metastatic cancers were positive for mammaglobin. In the current study, $65 \%$ of patients were positive for mammaglobin marker [29]. Saccani et al., in their study on CEA marker expression in 69 breast cancer patients, indicated an increase in expression of this marker in patients compared to healthy controls, which was in agreement with our finding [30]. Vizcarra et al., in their study used radioimmunoassay (RIA) for assessment of the expression of TPA, CA15.3 and CEA and reported an increase in expression of these markers in patients. Since the RIA method has lower sensitivity than the real-time PCR, studies using real-time 
PCR can better indicate the role of CEA as a suitable molecular biomarker for early detection of breast cancer [31]. Berois et al. in 2000 evaluated the expression of CK19, MUC1 and CEA genes using RT-PCR and demonstrated that expression of CK19 and CEA increased in patients with breast cancer, which was in line with the current results [32].

In conclusion, the current study was a preliminary study of breast cancer tumor markers, which introduced a diagnostic screening test for early detection of breast cancer at primary stages. Future studies with larger sample sizes are required to further confirm the current findings.

\section{References}

[1] Key, T.J., Verkasalo, P.K. and Banks, E. (2001) Epidemiology of Breast Cancer. The Lancet Oncology, 2, 133-140. https://doi.org/10.1016/S1470-2045(00)00254-0

[2] Mitchell, P.S., Parkin, R.K., Kroh, E.M., Fritz, B.R., Wyman, S.K., Pogosova-Agadjanyan, E.L., et al. (2008) Circulating microRNAs as Stable Blood-Based Markers for Cancer Detection. Proceedings of the National Academy of Sciences, 105, 10513-10518. https://doi.org/10.1073/pnas.0804549105

[3] Jemal, A., Siegel, R., Ward, E., Hao, Y., Xu, J., Murray, T., et al. (2008) Cancer Statistics, 2008. CA: A Cancer Journal for Clinicians, 58, 71-96.

https://doi.org/10.3322/CA.2007.0010

[4] Ban, K.A. and Godellas, C.V. (2014) Epidemiology of Breast Cancer. Surgical Oncology Clinics of North America, 23, 409-422.

https://doi.org/10.1016/j.soc.2014.03.011

[5] Group EBCTC (2005) Effects of Chemotherapy and Hormonal Therapy for Early Breast Cancer on Recurrence and 15-Year Survival: An Overview of the Randomised Trials. The Lancet, 365, 1687-717. https://doi.org/10.1016/S0140-6736(05)66544-0

[6] Ekizoglu, S., Muslumanoglu, M., Dalay, N. and Buyru, N. (2012) Genetic Alterations of the WWOX Gene in Breast Cancer. Medical Oncology, 29, 1529-1535. https://doi.org/10.1007/s12032-011-0080-0

[7] Stavros, A.T., Thickman, D., Rapp, C.L., Dennis, M.A., Parker, S.H. and Sisney, G.A. (1995) Solid Breast Nodules: Use of Sonography to Distinguish between Benign and Malignant Lesions. Radiology, 196, 123-134. https://doi.org/10.1148/radiology.196.1.7784555

[8] Chassevent, A., Jourdan, M.-L., Romain, S., Descotes, F., Colonna, M., Martin, P.-M., et al. (2001) S-Phase Fraction and DNA Ploidy in 633 T1T2 Breast Cancers. Clinical Cancer Research, 7, 909-917.

[9] Etzioni, R., Urban, N., Ramsey, S., McIntosh, M., Schwartz, S., Reid, B., et al. (2003) The Case for Early Detection. Nature Reviews Cancer, 3, 243-252. https://doi.org/10.1038/nrc1041

[10] Radpour, R., Barekati, Z., Kohler, C., Lv, Q., Bürki, N., Diesch, C., et al. (2011) Hypermethylation of Tumor Suppressor Genes Involved in Critical Regulatory Pathways for Developing a Blood-Based Test in Breast Cancer. PLoS One, 6, e16080. https://doi.org/10.1371/journal.pone.0016080

[11] Radpour, R., Barekati, Z., Kohler, C., Holzgreve, W. and Zhong, X.Y. (2009) New Trends in Molecular Biomarker Discovery for Breast Cancer. Genetic Testing and Molecular Biomarkers, 13, 565-571. https://doi.org/10.1089/gtmb.2009.0060 
[12] Srinivas, P.R., Kramer, B.S. and Srivastava, S. (2001) Trends in Biomarker Research for Cancer Detection. The Lancet Oncology, 2, 698-704. https://doi.org/10.1016/S1470-2045(01)00560-5

[13] Bhatt, A.N., Mathur, R., Farooque, A., Verma, A. and Dwarakanath, B. (2010) Cancer Biomarkers-Current Perspectives. Indian Journal of Medical Research, 132, 129-149.

[14] Bahrami, N., Gholami, M., Jamaati, H.R., Mohamadnia, A., Dargahi, H., Kazempour dizaji, M., Khosravi, A., Heshmatnia, J., Vahabi, P. and Bahrami, N.A. (2016) Expression of Two Essential mRNA Biomarker in the Peripheral Blood as Possible Biomarkers for Diagnosis of Non-Small Cell Lung Carcinoma. Minerva Pneumologica, 55, 31-36.

[15] Jamaati, H., Bahrami, N., Abniki, M., Tabarsi, P., Farzanegan, B., Doroudinia, A., et al. (2016) Real-Time RT-PCR Detection of HCN4 and ADAM8 Genes in Ventilator-Associated Pneumonia Patients Hospitalized in Intensive Care Unit. Journal of Cellular and Molecular Anesthesia, 1, 163-167.

[16] Khare, P.D., Shao-Xi, L., Kuroki, M., Hirose, Y., Arakawa, F., Nakamura, K., et al. (2001) Specifically Targeted Killing of Carcinoembryonic Antigen (CEA)-Expressing Cells by a Retroviral Vector Displaying Single-Chain Variable Fragmented Antibody to CEA and Carrying the Gene for Inducible Nitric Oxide Synthase. Cancer Research, 61, 370-375.

[17] Ebeling, F., Stieber, P., Untch, M., Nagel, D., Konecny, G., Schmitt, U., et al. (2002) Serum CEA and CA 15-3 as Prognostic Factors in Primary Breast Cancer. British Journal of Cancer, 86, 1217-1222. https://doi.org/10.1038/sj.bjc.6600248

[18] Zehentner, B.K. and Carter, D. (2004) Mammaglobin: A Candidate Diagnostic Marker for Breast Cancer. Clinical Biochemistry, 37, 249-257. https://doi.org/10.1016/j.clinbiochem.2003.11.005

[19] Fleming, T.P. and Watson, M.A. (2000) Mammaglobin, a Breast-Specific Gene, and Its Utility as a Marker for Breast Cancer. Annals of the New York Academy of Sciences, 923, 78-79. https://doi.org/10.1111/j.1749-6632.2000.tb05521.x

[20] Bossolasco, P., Ricci, C., Farina, G., Soligo, D., Pedretti, D., Scanni, A., et al. (2002) Detection of Micrometastatic Cells in Breast Cancer by RT-pCR for the Mammaglobin Gene. Cancer Detection and Prevention, 26, 60-63. https://doi.org/10.1016/S0361-090X(02)00009-0

[21] Grünewald, K., Haun, M., Urbanek, M., Fiegl, M., Müller-Holzner, E., Gunsilius, E., et al. (2000) Mammaglobin Gene Expression: A Superior Marker of Breast Cancer Cells in Peripheral Blood in Comparison to Epidermal-Growth-Factor Receptor and Cytokeratin-19. Laboratory Investigation, 80, 1071-1077. https://doi.org/10.1038/labinvest.3780112

[22] Mohamadnia, A., Karimi, S., Yadegar Azari, R., Naji, S.A., Khosravi, A., Bahrami, N., et al. (2016) Expression of CK19 Gene in Patients with Lung Cancer and Its Comparison with Carcinoembryonic Antigen in Peripheral Blood. Journal of Payavard Salamat, 9, 459-468.

[23] Jemal, A., Siegel, R., Ward, E., Hao, Y., Xu, J. and Thun, M.J. (2009) Cancer Statistics, 2009. CA: A Cancer Journal for Clinicians, 59, 225-249. https://doi.org/10.3322/caac.20006

[24] Ludwig, J.A. and Weinstein, J.N. (2005) Biomarkers in Cancer Staging, Prognosis and Treatment Selection. Nature Reviews Cancer, 5, 845-856. https://doi.org/10.1038/nrc1739 
[25] Karimi, S., Mohamadnia, A., Nadji, S.A., Yadegarazari, R., Khosravi, A., Bahrami, N., et al. (2015) Expression of Two Basic mRNA Biomarkers in Peripheral Blood of Patients with Non-Small Cell Lung Cancer Detected by Real-Time rt-PCR, Individually and Simultaneously. Iranian Biomedical Journal, 19, 17.

[26] Ghadimi, K., Bahrami, N., Fathi, M., Farzanegan, B., Naji, T., Emami, M., et al. (2017) Diagnostic Value of LunX mRNA and CEA mRNA Expression in Pleural Fluid of Patients with Non-Small Cell Lung Cancer. Minerva Pneumologica, 56.

[27] Karimi, S., Bahrami, N., Sharifi, K., Daustany, M., Baghbani-Arani, F., Kazempour, M., et al. (2017) Investigating Gene Expression Level of MUC1 and CEA in Pleural Fluid of NSCLC Lung Cancer Patients with Real-Time RT-PCR Method. Minerva Pneumologica, 56, 18-24.

[28] Moshref Behzad, N., Bahrami, N., Farzanegan, B., Fathi, M., Zareh Karizi, S. and Mohamadnia, A. (2017) Expression of CK19-mRNA and CEA-mRNA Biomarkers in Pleural Fluid of Patients with Non-Small Cell Lung Cancer. Minerva Pneumologica, 56.

[29] Watson, M.A., Dintzis, S., Darrow, C.M., Voss, L.E., DiPersio, J., Jensen, R., et al. (1999) Mammaglobin Expression in Primary, Metastatic, and Occult Breast Cancer. Cancer Research, 59, 3028-3031.

[30] Saccani, J.G., Bombardieri, E., Fontanesi, M., Becchi, G., Bocchi, P., Palli, D., et al. (1988) Plasma and Tissue CEA and TPA Markers in Operable Breast Cancer. Preliminary Results. Tumori, 74, 681-688.

[31] Vizcarra, E., Lluch, A., Cibrian, R., Jarque, F. and Garcia-Conde, J. (1994) CA15.3, CEA and TPA Tumor Markers in the Early Diagnosis of Breast Cancer Relapse. Oncology, 51, 491-496. https://doi.org/10.1159/000227391

[32] Berois, N., Varangot, M., Aizen, B., Estrugo, R., Zarantonelli, L., Fernandez, P., et al. (2000) Molecular Detection of Cancer Cells in Bone Marrow and Peripheral Blood of Patients with Operable Breast Cancer. Comparison of CK19, MUC1 and CEA using RT-PCR. European Journal of Cancer, 36, 717-723. https://doi.org/10.1016/S0959-8049(99)00338-X 